\title{
"El AGUAJAL": EL BOSQUE DE LA VIDA EN LA AMAZONÍA PERUANA
}

\author{
Julio Ruiz-Murrieta*,1 y Jeanine Levistre-Ruiz² \\ ${ }^{1}$ Universidad Científica del Perú, Avda. Abelardo Quiñones Km 2.5 Iquitos, Perú. \\ 2Organización de las Naciones Unidas para la Educación, la Ciencia y la Cultura - UNESCO. \\ e-mail: jruiz@ucp.edu.pe
}

\begin{abstract}
RESUMEN
En este artículo se presenta el ecosistema forestal más remarcable de la Amazonía peruana: el "Aguajal", llamado por los pueblos indígenas el "bosque de la vida", de donde ellos extraen una gran diversidad de productos para su alimentación, medicina, construcción, ritos y arte tradicional, entre otros. Se presenta recomendaciones para su uso sostenible, el mismo que se sustenta sólidamente en la viabilidad económica. Sin embargo, se valoran también los aspectos sociales, culturales y ecológicos del aguajal, los mismos que reflejan los múltiples usos que hacen de este ecosistema las poblaciones indígenas. La investigación se llevó a cabo en la región Loreto dentro del marco del Programa El Hombre y la Biosfera de la UNESCO.
\end{abstract}

Palabras Claves: aguajal, ecosistema, pueblos indígenas, manejo, cultura.

\section{"EL AGUAJAL": THE FOREST OF LIFE IN THE PERUVIAN AMAZON}

\begin{abstract}
We present here the most remarkable forest ecosystem of the Peruvian Amazon: "the Aguajal", called by the indigenous peoples the "forest of life". From this ecosystem indigenous peoples harvest a great diversity of forest products for food, construction, medicine, rites, and traditional art among others. This article, presents also recommendations for sustainable management of aguajales, which is bases on its economic viability. Nevertheless, we present also, ecological, social and cultural values, which reflect the multiple uses that the indigenous populations make from this ecosystem. The research was done within the framework of the UNESCO Man and Biosphere Program (MAB Program).
\end{abstract}

Key words: aguajal, ecosystem, indigenous peoples, management, culture. 


\section{INTRODUCCIÓN}

Los aguajales son ecosistemas forestales húmedos cubiertos de una extraordinaria vegetación pantanosa, donde la planta dominante es el aguaje. El aguaje es un árbol que pertenece a la familia de las palmeras. Las especies más importantes son Mauritia flexuosa, Mauritia vinífera, Mauritiella peruviana (aguajillo). La extensión de estos aguajales naturales en la planicie amazónica del Perú es importante. Un ejemplo de estos ecosistemas se da en la depresión Ucamara (Ucayali-Marañón), en la región Loreto, cuya capital es Iquitos, donde la superficie de los aguajales alcanza cerca de 4 millones de hectáreas ( $10 \%$ de la superficie de la región) 7,8 .

En la región Loreto, así como en el resto de las áreas rurales de la Amazonía peruana, la población depende de los aguajales para su alimentación, salud, construcción de viviendas, elaboración de utensilios, etc., lo cual son ejemplos del valor de estos ecosistemas. Los pobladores de la Amazonía peruana, aprovechan diversos productos de los aguajales: madera, hojas de palmeras y otros productos diferentes a la madera, así como animales silvestres que habitan en estos ecosistemas. Transforman las hojas del aguaje en crisnejas, ahúman la carne del monte, transforman algunas semillas, fibras, cortezas y raíces en artesanía, lo cual implica muy poco valor agregado. Solamente la ciudad de Iquitos, consume 20 toneladas de frutos de aguaje por día. La producción anual del fruto es aproximadamente de 100 a $200 \mathrm{~kg}$ por planta. ${ }^{2}$

Al problema de escaso valor agregado se suma el de la degradación de estos ecosistemas, sobre todo de la palmera del aguaje, que han sido sobre explotados por años o aprovechados con técnicas destructivas de cosechas. El resultado final de esta presión cada vez más fuerte sobre estos recursos forestales, es la degradación de los ecosistemas y como consecuencia la profundización y extensión creciente de la pobreza rural, como es el caso de la región Loreto.

Romper el círculo vicioso de pobreza y deterioro de los recursos forestales implica poner en marcha una política, donde se vea a la Amazonía como un ecosistema habitado por una diversidad de especies de plantas y animales, así como por poblaciones indígenas y ribereñas de las cuales es necesario conocer su cultura y su relación con el bosque. Implica también emprender proyectos de investigación y desarrollo que vayan de la mano con el desarrollo sostenible y orientado a mejorar las condiciones de vida del poblador rural. Implica también que se promulguen leyes consultadas con las poblaciones locales, que generen riqueza para las poblaciones que habitan en los bosques, respetando siempre las convenciones internacionales como la Convención sobre la Diversidad Biológica y la Convención 169 de la OIT. Este artículo presenta nuevos conocimientos sobre los aguajales, considerado por la UNESCO como ecosistemas remarcables y por los indígenas de la Amazonía peruana como el bosque de la vida.

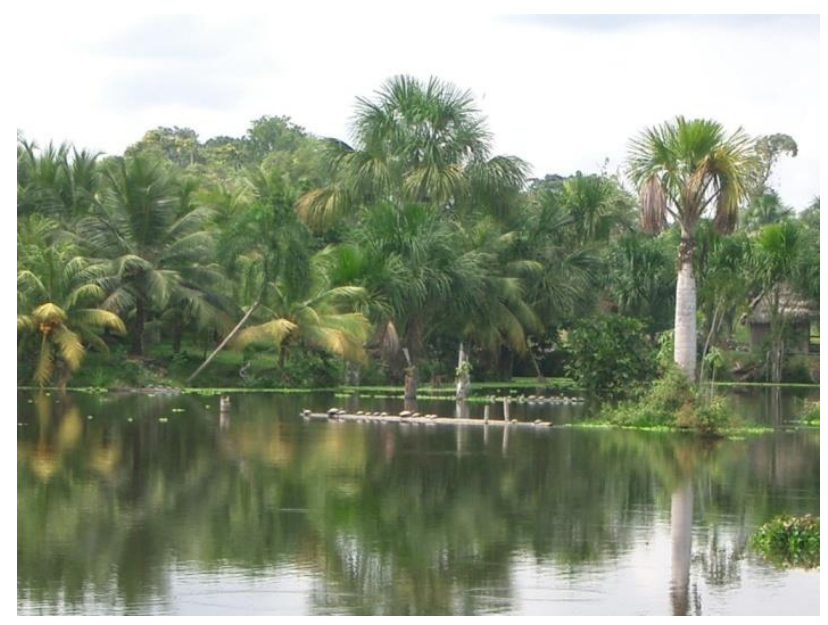

Foto 1: Aguajal: Ecosistema forestal remarcable de la Amazonía peruana 


\section{MATERIALES Y METODOS}

El presente trabajo de investigación se desarrolló en diversas zonas de la región Loreto en la Amazonía peruana durante el periodo 1985-1995, dentro del marco del Programa el Hombre y la Biosfera de la UNESCO y del Programa Mundial de Bosques de la Unión Mundial para la Conservación de la Naturaleza (UICN). El levantamiento de información se realizó en el campo, realizándose inventarios forestales en aguajales de la cuenca del Marañón con el apoyo de materos indígenas de la etnia Kukama Kukamira. Se buscó información bibliográfica en libros y revistas especializadas y se realizaron encuestas antropológicas a 127 personas de las etnias Yagua, Kukama Kukamira y Quichuas del Napo.

\section{RESULTADOS Y DISCUSIONES}

\section{Valor ecológico del aguajal}

El aguajal es el ecosistema más importante y el menos conocido en la Amazonía peruana. Según los últimos estudios realizados a partir de imágenes satelitales se estima que los aguajales ocupan un área de 6 a 8 millones de hectáreas en toda la Amazonía peruana (IIAP, 1988). De este valor aproximadamente 2.15 millones de hectáreas son aguajales puros, donde la densidad es muy elevada, alcanzando desde 180 plantas por hectárea en el río Itaya cerca de Iquitos hasta 351 plantas por hectárea en la cuenca del Huallaga. ${ }^{10}$

Los aguajales son, entre otros, el hábitat natural de diversas especies de plantas de importancia económica, alimenticia y cultural tales como el ungurahui (Jessenia bataua), el huasaí (Euterpe precatoria), la chonta (Euterpe oleracea), el sinamillo (Oenocarpus mapora), la huacrapona (Iriartea deltoidea), la poloponta (Elais oleifera H.B.K.), la yarina (Phytelephas microcarpa), el copal (Protium $s p$.) y el charichuelo (Rheedia $s p$.). Un inventario realizado en un aguajal en la región del Ucayali muestra un total de 18 especies de palmeras por hectárea entre ellas 4 especies productoras de alimentos de alto valor nutricional, las mismas que juegan además un rol muy importante en el régimen alimenticio de las poblaciones rurales y urbanas. ${ }^{3,4}$

Igualmente, los aguajales constituyen una fuente de alimentos de origen animal que son consumidos por la población indígena de las riberas de la Amazonía. Tal es el caso del Suri, larva del coleóptero (Rynchophorus palmarum) muy rico en grasas y proteínas, que se desarrollan en los troncos muertos del aguaje. Igualmente encontramos en el aguajal mamíferos, reptiles, insectos y moluscos que son utilizados en la alimentación humana.

Finalmente, el gran valor ecológico de los aguajales reside en que es un medio natural de autodefensa contra la destrucción del bosque por el hombre. En efecto, los colonos perciben al aguajal como un medio hostil, ya que está inundado permanentemente por agua negras y es el hábitat de numerosos insectos y reptiles venenosos, entre ellos, el aguaje machaco (Bothrops bileneatus), la anaconda (Eunectes murinus), el lagarto negro (Melanosuchus niger) y miles de sanguijuelas (Ver Tablas 1 y 2 ) 
Tabla 1. Principales productos vegetales del Aguajal

\begin{tabular}{|c|c|c|c|c|c|c|c|c|c|}
\hline & \multicolumn{6}{|c|}{ Utilización } & \multicolumn{3}{|c|}{ Valor } \\
\hline & Alimentación & Comercio & Construcción & $\begin{array}{l}\text { Medicina } \\
\text { y Magia }\end{array}$ & Tecnológica & $\begin{array}{l}\text { La } \\
\text { caza }\end{array}$ & Económico & Cultural & Alimenticio \\
\hline $\begin{array}{l}\text { Aguaje } \\
\text { (Mauritia } \\
\text { flexuosa) }\end{array}$ & & & & & & & & & \\
\hline Frutos & + & + & - & - & + & + & + & + & + \\
\hline Hojas & - & + & + & - & + & - & + & + & - \\
\hline Troncos & - & - & + & - & - & - & + & + & - \\
\hline $\begin{array}{l}\text { Tronco } \\
\text { muerto } \\
\text { Aguaje } \\
\text { Mauritia } \\
\text { vinífera }\end{array}$ & + & + & - & + & - & - & + & + & + \\
\hline Frutos & + & + & - & - & + & + & + & + & + \\
\hline Hojas & - & + & + & - & + & - & + & + & - \\
\hline Tronco & - & - & + & - & - & - & + & + & - \\
\hline $\begin{array}{l}\text { Tronco } \\
\text { muerto } \\
\text { Inflorescencia } \\
\text { Aguajillo } \\
\text { Mauritiella } \\
\text { peruviana }\end{array}$ & + & + & - & + & - & - & + & + & + \\
\hline Frutos & + & + & - & - & - & + & + & + & + \\
\hline Hojas & - & + & - & - & + & - & + & + & - \\
\hline $\begin{array}{l}\text { Tronco } \\
\text { Ungurahui } \\
\text { Jessenia } \\
\text { bataua }\end{array}$ & - & - & + & - & - & - & - & - & - \\
\hline Frutos & + & + & - & - & - & + & + & + & + \\
\hline $\begin{array}{l}\text { Aceite de los } \\
\text { frutos } \\
\text { Huasaí } \\
\text { Euterpe } \\
\text { precatoria }\end{array}$ & - & + & - & + & + & - & + & + & - \\
\hline Frutos & + & + & - & - & - & + & + & + & - \\
\hline $\begin{array}{l}\text { Corazón de la } \\
\text { palmera }\end{array}$ & + & + & - & - & - & - & + & + & + \\
\hline $\begin{array}{l}\text { Inflorescencia } \\
\text { Sinamillo } \\
\text { Oenecarpus } \\
\text { mapora }\end{array}$ & - & - & - & - & + & - & - & + & - \\
\hline $\begin{array}{l}\text { Frutos } \\
\text { Huacrapona } \\
\text { Iriartea } \\
\text { deltoidea }\end{array}$ & + & + & - & - & - & + & + & + & + \\
\hline Troncos & - & + & + & - & - & - & + & + & - \\
\hline $\begin{array}{l}\text { Corazón de la } \\
\text { palmera } \\
\text { Poloponta } \\
\text { Elaeis } \\
\text { oleífera }\end{array}$ & + & + & - & - & - & - & + & + & + \\
\hline $\begin{array}{l}\text { Aceite de los } \\
\text { frutos } \\
\text { Yarina } \\
\text { Phytelephas } \\
\text { microcarpa }\end{array}$ & + & - & - & - & - & - & + & + & + \\
\hline Frutos & + & + & - & - & - & - & + & + & + \\
\hline Hojas & - & + & + & - & - & - & + & + & - \\
\hline $\begin{array}{l}\text { Copal } \\
\text { Protium sp. }\end{array}$ & & & & & & & & & \\
\hline Frutos & + & + & - & - & - & + & + & + & + \\
\hline Resina & - & + & + & - & + & - & + & + & - \\
\hline Corteza & - & - & - & - & + & - & - & + & - \\
\hline Troncos & - & + & + & - & - & - & + & + & + \\
\hline $\begin{array}{l}\text { Charichuelo } \\
\text { Rhedia Sp. }\end{array}$ & & & & & & & & & \\
\hline Frutos & + & + & - & - & - & + & + & + & + \\
\hline Látex & - & + & - & + & - & - & - & + & - \\
\hline Troncos & - & - & + & - & - & - & - & - & - \\
\hline
\end{tabular}

Cienc. amaz., Vol. 1, No.1, 2011 
Tabla 2. Principales productos animales del Aguajal

\begin{tabular}{|c|c|c|c|c|c|c|c|c|c|}
\hline & \multicolumn{6}{|c|}{ Utilización } & \multicolumn{3}{|c|}{ Valor } \\
\hline & Alimentación & Comercio & Construcción & $\begin{array}{l}\text { Medicina } \\
\text { y Magia }\end{array}$ & Tecnológica & $\begin{array}{c}\text { La } \\
\text { caza }\end{array}$ & Económico & Cultural & Alimenticio \\
\hline $\begin{array}{l}\text { Suri } \\
\text { Rynchopharus } \\
\text { palmarum }\end{array}$ & & & & & & & & & \\
\hline Carne & + & + & - & - & - & - & + & + & + \\
\hline $\begin{array}{l}\text { Aceite } \\
\text { Otorongo } \\
\text { Pantera onca }\end{array}$ & - & - & - & + & - & - & - & + & - \\
\hline $\begin{array}{l}\text { Piel } \\
\text { Anaconda } \\
\text { Eunectes } \\
\text { murinus }\end{array}$ & - & + & - & - & - & - & + & - & - \\
\hline Piel & - & + & - & - & - & - & + & - & - \\
\hline $\begin{array}{l}\text { Grasa } \\
\text { Lagarto Blanco } \\
\text { Caiman } \\
\text { silevopus } \\
\text { Carne }\end{array}$ & - & - & - & + & - & - & + & + & - \\
\hline Piel & + & + & - & - & - & - & + & + & + \\
\hline Grasa & - & + & - & - & - & - & + & - & - \\
\hline $\begin{array}{l}\text { Lagarto Negro } \\
\text { Melanosuchus } \\
\text { niger } \\
\text { Piel }\end{array}$ & - & + & - & + & - & - & + & + & - \\
\hline $\begin{array}{l}\text { Boa } \\
\text { Boa } \\
\text { constrictor }\end{array}$ & - & + & - & - & - & - & + & - & - \\
\hline Piel & - & + & - & - & - & - & + & - & - \\
\hline $\begin{array}{l}\text { Grasa } \\
\text { Sachavaca } \\
\text { Tapirus } \\
\text { terrestris }\end{array}$ & - & + & - & + & - & - & + & - & - \\
\hline $\begin{array}{l}\text { Carne } \\
\text { Ronsoco } \\
\text { Cuniculus paca } \\
\text { Carne }\end{array}$ & + & + & - & - & - & - & + & + & + \\
\hline $\begin{array}{l}\text { Añuje } \\
\text { Dasyprocta sp. } \\
\text { Carne } \\
\text { Sajino Tayassu }\end{array}$ & + & + & - & - & - & - & + & + & + \\
\hline $\begin{array}{l}\text { tajacu } \\
\text { Carne } \\
\text { Piel } \\
\text { Huangana }\end{array}$ & + & + & - & - & - & - & + & + & + \\
\hline Tayassu pecari & + & + & - & - & - & - & + & + & + \\
\hline $\begin{array}{l}\text { Carne } \\
\text { Piel } \\
\text { Churo } \\
\text { Strophoecheilu } \\
\text { papelairianus }\end{array}$ & - & + & - & - & - & - & + & - & - \\
\hline $\begin{array}{l}\text { Carne } \\
\text { Diversos } \\
\text { Pájaros }\end{array}$ & + & + & - & - & - & - & + & + & + \\
\hline Carne & + & - & - & - & - & - & - & + & + \\
\hline Plumaje & - & - & - & + & + & - & - & + & - \\
\hline
\end{tabular}




\section{Valor cultural del aguajal}

\section{El aguajal en el mundo de los pueblos indígenas}

En la Amazonía peruana las manifestaciones culturales de los diversos pueblos indígenas, entre ellas las creencias, los comportamientos, los ritos, los mitos, las costumbres, los conocimientos tradicionales, entre los más importantes, representan valiosos elementos que es necesario conocerlos e integrarlos para asegurar el manejo sostenible de los recursos forestales.

Para los Yagua y otros pueblos indígenas que pueblan la Amazonía peruana el aguajales considerado como la madre de la selva, que crea el aguaje, la fruta más deliciosa del bosque. También del árbol de aguaje ellos extraen diversos alimentos de origen animal y vegetal, así como materiales para la construcción, la artesanía, la caza, la pesca, la medicina y la magia. Los Yagua, creen que el otorongo, la anaconda, la lechuza, el lagarto negro y el hombre son hijos del aguajal. Estas especies animales simbolizan ante todo los espacios acuáticos, terrestres y celestiales, que corresponden al aguajal. El otorongo encarna a un curandero muerto y a él le atribuyen casi todos los poderes de la reencarnación. El otorongo es también el amo de selva, del eco, de los animales silvestres y de los tambores que llaman a la vida. La anaconda vive en el aguajal y allí caza sus presas. La anaconda simboliza el inconsciente colectivo, el saber y las técnicas de caza, es el amo de la sabiduría y el dueño de todos los secretos del aguajal. La lechuza, parada sobre las ramas del aguaje, árbol cósmico, cura como un remedio todos los males. Es sobre este aspecto medicinal-mágico que le honran los Yagua y otros pueblos indígenas: el curandero frota la cabeza del enfermo con una pluma de lechuza, la misma que absorbe el mal y cura al enfermo. Los Yagua no tumban nunca el árbol femenino de aguaje para coger sus frutos. Esto debido a la creencia de que el lagarto negro, hijo del aguajal, devora todo, sobre todo a los hombres que tumban el árbol femenino del aguaje, que proporciona los frutos. Para los Yagua, el árbol del aguaje es símbolo de inmortalidad, como el sol renaciente que realiza el ciclo cósmico. El árbol del aguaje y sus grandes bellas hojas en abanico, con sus raíces en el mundo infernal, pero esparcidos en el cielo, indican para los Yagua el crecimiento en diversas direcciones y la manifestación del espacio. Es a través de las hojas del aguaje hembra, llamada mama aguaje, que el alma puede remontar al cielo. Este árbol (el aguaje) se integra en la creencia de la inmortalidad del alma de los Yagua. ${ }^{7}$

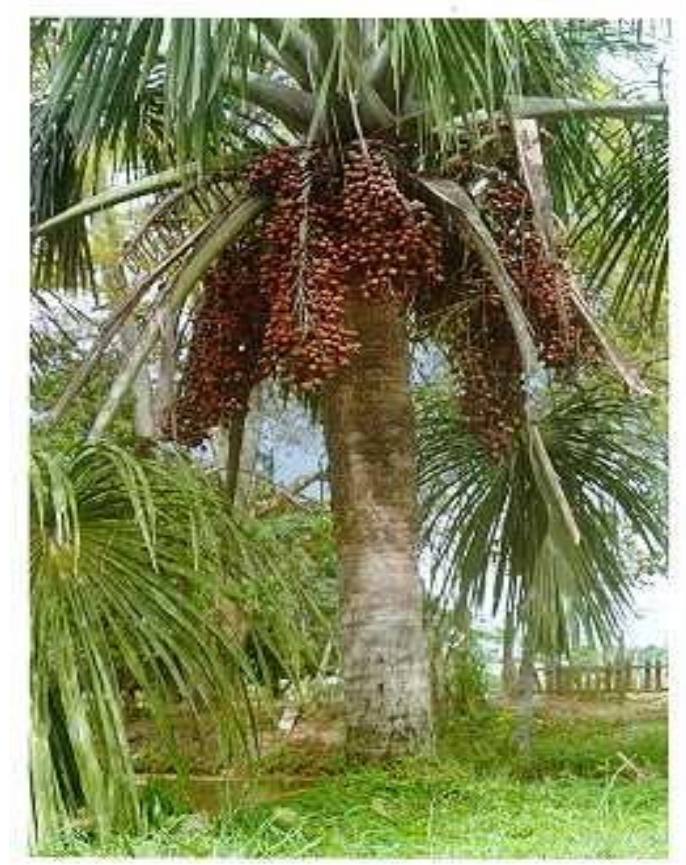

Foto 2. El aguaje se integra en la creencia de la inmortalidad del alma de los Yagua. 
Las diversas técnicas de caza que existen en la Amazonía peruana han sido elaboradas a partir de los conocimientos que tienen los pueblos indígenas de la taxonomía, la biología y la ecología de las plantas y los animales silvestres. Los indígenas de la Amazonía utilizan en los aguajales la técnica llamada "bebedores naturales", que consiste en poner jaulas en los pies de los árboles que sirven de alimentación a muchos animales según la época de fructificación. Por ejemplo, en los pies del árbol del aguaje y del aguajillo se instalan jaulas en los meses de julio y agosto para la caza del majaz (Cuniculus paca) cuya carne es muy apreciada. La caza de mamíferos mayores, entre ellos el sajino (Tayassu tajacu), la huangana (Tayassu albirostis) y la sacha vaca (Tapirus terrestres), se efectúa también en los aguajales, los mismos que son bebederos para estos animales ${ }^{1}$. Los aguajales constituyen igualmente un medio natural ideal para cazar otros animales.

Los Yagua, así como la gran mayoría de los pueblos indígenas de la Amazonía peruana, comen el mesocarpio del fruto, el mismo que constituye un alimento regular de su dieta. Pero el aguaje no solo es un alimento. El árbol es también utilizado por sus fibras. Con ellas los Yagua construyen sus vestimentas, artículos domésticos y objetos de artesanía tales como sacos, canastas, etc. A partir del peciolo del aguaje muchas comunidades indígenas construyen esteras que sirven de división en las casas, así como alfombras rusticas para dormir.

\section{El aguajal en el mundo urbano de la Amazonía.}

No es exagerado decir que el aguaje es la fruta que tiene el mayor valor cultural en la región. El consumo del aguaje es importante, tanto por la cantidad de productos que se derivan del fruto, como por el hecho de que es la fruta preferida por la población urbana de la Amazonía peruana, especialmente por las mujeres, por su sabor agradable y por su valor simbólico.

Es por esto que la fruta del aguaje ocupa un lugar importante en la mitología de la ciudad de Iquitos, capital de la Amazonía peruana, donde se consume aproximadamente 20 toneladas de aguaje por día. En Iquitos, el aguaje es considerado la fruta del amor y la feminidad. Comer algunas frutas diarias es una necesidad para la mayoría de mujeres de Iquitos. Para ellas, según la creencia, el aguaje les embellece el cuerpo y las hace más mujeriles debido a su contenido de hormonas femeninas. Es a causa de este valor simbólico que las mujeres consumen más frutas maduras que los hombres. En Iquitos se acostumbra comer como postre el aguaje con sal.

La lengua española que se habla en la Amazonía peruana, de entonación dulce y agradable, ha enriquecido su vocabulario gracias al aporte del aguaje. Así, hay palabras nuevas como aguajal (ya descrito), curichi (pasta de aguaje mezclado con agua y azúcar y congelados en pequeñas bolsitas de plástico), curichero (vendedor de curichi), aguajera (vendedora de aguaje), shambo (aguaje con piel rojiza), ponguete (aguaje con piel amarilla), piquicho (aguaje con la semilla deformada). Estas palabras son sólo algunos ejemplos que muestran la contribución del aguaje a la cultura oral de la región. Finalmente, tanta es la adoración de la población amazónica por el aguaje que en los años 90 apareció la canción el aguajal que describe los amores que salen del aguajal.

\section{Valor cultural de otros productos del aguajal.}

El valor cultural de otros productos que se extraen del aguajal ocupa un lugar importante en el saber y en el conocimiento tradicional de las poblaciones rurales y urbanas de la Amazonía peruana. Por ejemplo, la inflorescencia del aguaje y del huasai se usa para hacer escobas y juguetes para los niños. El aceite del ungurahui se usa como medicina tradicional eficaz para combatir la calvicie y para embellecer el pelo. La sabia del tronco del sinamillo es un remedio contra la fiebre intestinal. Los troncos de la huacrapona son muy apreciados para la construcción de planchas en las casas tradicionales.

Las hojas secas de la yarina son utilizadas para la construcción de techos en las casas populares y el endosperma maduro del fruto es utilizado como marfil vegetal para la fabricación de botones y otras artesanías de valor. 
La resina del copal es utilizada como barniz y como cola. El ungüento que se extrae del copal es un remedio para las enfermedades de los aparatos respiratorio y urinario. La lista de los productos forestales no leñosos que se extraen del aguajal y que se usan en la alimentación, la construcción, la medicina, la magia, la artesanía tradicional y en el comercio es tan larga, la misma que se resume en las tablas 1 y 2 .

\section{Valor económico del aguajal}

Entre los numerosos productos que se extraen del bosque, el fruto del aguaje es el producto forestal, además de la madera, más importante en la economía de la Amazonía peruana. Social y económicamente el fruto del aguaje, en sus diferentes formas y procesamientos, está asociado con los pobres y las mujeres. El aguaje es fuente de empleo y de ingresos para un porcentaje elevado de la población sobre todo femenina. Las aguajeras (mujeres pobres que venden aguajes en las esquinas de las calles) y los aguajeros (niños pobres que desarrollan igual tarea) son típicos del paisaje urbano y de los mercados de la ciudad de Iquitos. Para estas personas la venta del aguaje es el único medio de subsistencia. ${ }^{5}$

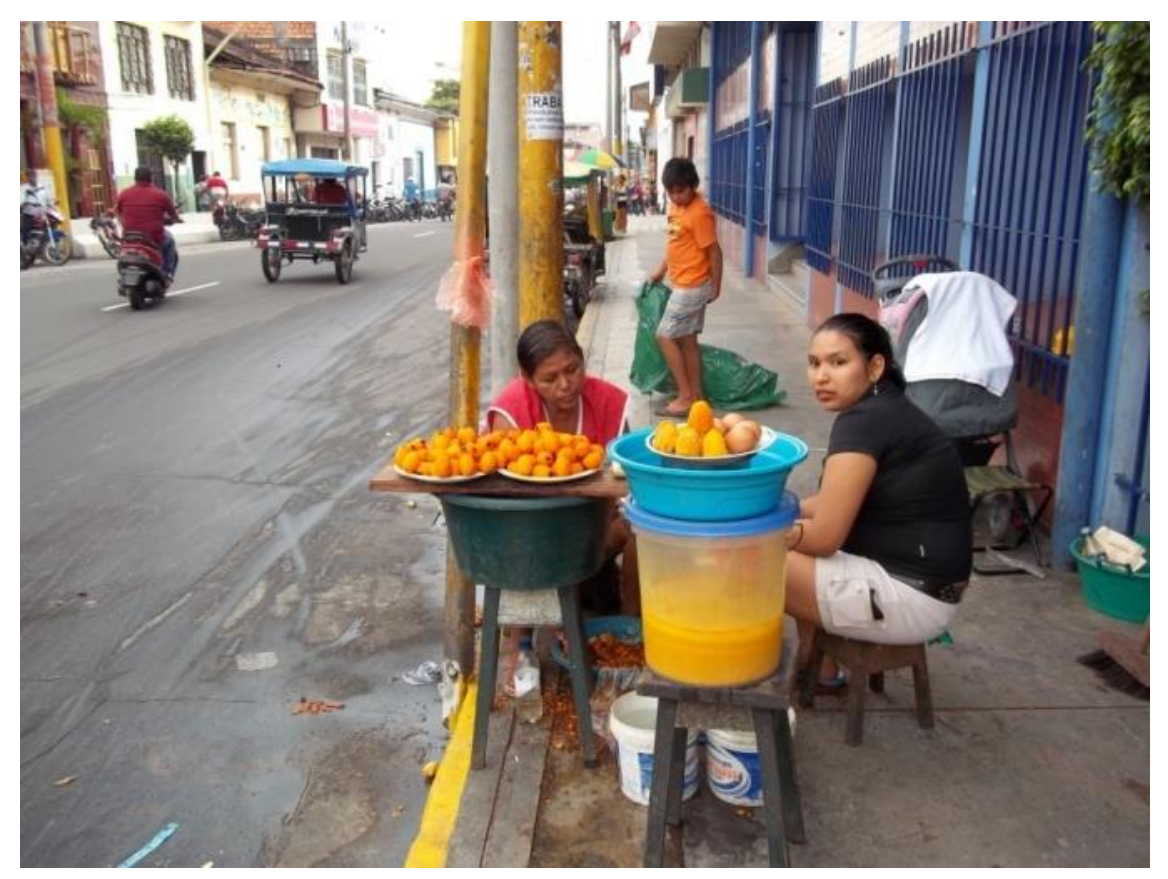

Foto 3. Vendedoras de aguaje en la ciudad de Iquitos.

El número de personas involucradas en la extracción, el transporte, la transformación, el comercio y el consumo del aguaje es muy elevado. El aguaje ha creado una serie de actividades especializadas que requieren de una tecnología rústica, las mismas que están representadas por los extractores, los transportistas, los mayoristas, los minoristas, las aguajeras, los vendedores de masa de aguaje, los comerciantes de helados, de chupetes y de curichis de aguaje, las vendedoras de aguajinas, etc.

Nuestra investigación ha estimado que sólo en la ciudad de Iquitos cerca de 2000 personas (la mayoría de ellos con familias numerosas) obtienen sus beneficios únicamente de la venta del aguaje y les representa aproximadamente una ganancia de 80 a 100 soles por día.

Teniendo en consideración los diferentes productos que tienen como base el fruto del aguaje podemos afirmar que existe en la Amazonía peruana una pequeña industria significativa de transformación del aguaje. 


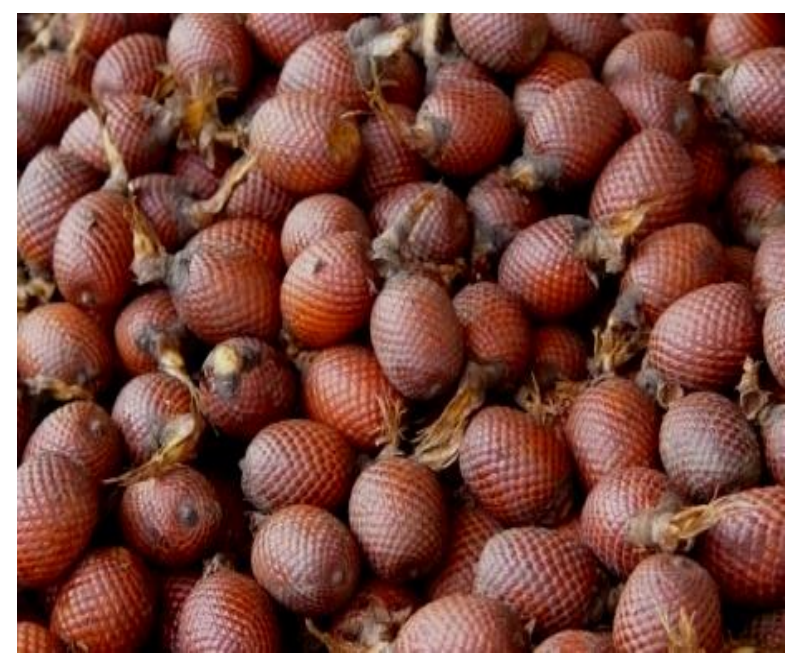

Foto 4. Fruto del aguaje de venta en los mercados de Iquitos.

\section{CONCLUSIONES Y RECOMENDACIONES}

El manejo de los aguajales, así como del bosque tropical húmedo no podrá realizarse si no se toman en consideración los valores sociales y culturales, así como los económicos y ecológicos de estos recursos.

Esto implica:

- Que los planificadores y los administradores del desarrollo reconozcan que los sistemas forestales remarcables y estratégicos tienen una importancia grande para las comunidades, principalmente indígenas. Este es el caso de los aguajales.

- Que se valoren otros productos del bosque además de la madera, principalmente alimentos.

- Admitir que la capacidad de los que realizan el manejo del bosque es limitada y se necesita por tanto el conocimiento tradicional de las comunidades indígenas y mestizas ribereñas que hasta ahora ha estado discriminado y no utilizado.

- Reconocer la experiencia adquirida por las organizaciones de grupos tradicionales en la planificación, aplicación, gestión y desarrollo de productos forestales no leñosos, así como en la explotación comercial.

La valoración de los ecosistemas forestales en la Amazonía peruana ha avanzado incipientemente en sus aspectos ecológicos y económicos. Los aspectos sociales y culturales recién están comenzando a ser valorados.

Un equipo conformado por la Universidad Científica del Perú, dentro del marco del Programa el Hombre y la Biosfera (MAB) de la Unesco, está estudiando la ecología de los aguajales, la importancia del aguaje en la economía regional, así como la localización y la extensión de los aguajales usando técnicas de teledetección. Estos estudios están poniendo en evidencia que este ecosistema puede ser una alternativa para aliviar los problemas alimenticios y socioeconómicos de la región Loreto debido a su enorme potencial de uso.

El mismo equipo está realizando diversos estudios culturales sobre la utilización tradicional de productos forestales no leñosos en la Amazonía peruana donde se muestra la viabilidad del manejo sostenible en los aspectos culturales económicos y ecológicos de estos productos. 
Esos estudios están mostrando que las familias de plantas más utilizadas por las poblaciones tradicionales son las palmeras, las anonáceas y las lauráceas, utilizadas principalmente como material de construcción tradicional en el comercio, en la alimentación y en la medicina.

Gracias a estos estudios están apareciendo nuevas estrategias de manejo en las reservas comunitarias donde se favorece la utilización sostenible de recursos naturales, tales como la pesca, la caza y la agroforestería.

Las reservas comunitarias de la Amazonía peruana a diferencia de las reservas extractivas en el Brasil se están desarrollando sin violencia social. ${ }^{9}$ En ellas se practica una extracción más diversificada de productos forestales no leñosos dando prioridad a la satisfacción de la necesidad de las poblaciones locales. Estas estrategias están fundamentadas bajo el concepto de "el bosque como fuente de alimento"; los mejores ejemplos son las reservas comunitarias de Yanesha y de Tamishacu-Tahuayo. Esta última es una reserva de ribereños y es un ejemplo de participación de la comunidad local en la planificación, en el control y la gestión de la reserva, utilizando múltiples productos del bosque y respetando y valorando la cultura amazónica.

Un ejemplo de este tipo de organizaciones es la Federación de Campesinos e Indígenas de Loreto (FEDECANAL). El manejo de las reservas existentes es ahora una cogestión entre el Gobierno Regional de Loreto, FEDECANAL y 44 comunidades campesinas e indígenas. El objetivo de FEDECANAL es transformar estas comunidades en administradores de sus propios bosques. ${ }^{6}$

Actualmente el obstáculo más importante para el manejo sostenible de los aguajales está en la utilización de técnicas destructivas de los frutos, los mismos que se hacen tumbando los árboles, lo que produce progresivamente la disminución del potencial genético y comercial de los aguajales. En la cuenca del rio Nanay diversas comunidades ribereñas están utilizando nuevas técnicas no destructivas para cosechar los aguajales. Se recomienda profundizar las investigaciones sobre prácticas tradicionales de manejo de ecosistemas forestales, incluyendo los "varillales", que vienen realizando las poblaciones ribereñas e indígenas de la cuenca del rio Nanay, cuyos resultados son hasta la actualidad muy alentadores.

\section{REFERENCIAS}

(1) Bodmer, R.E. et al. (1988). Estudios y manejo de los pecarís (Tayassu tayacu y T. pecari) en la Amazonía peruana. Matero. pp. 18-25. Iquitos. UNAP.

(2) IIAP (1988). La explotación del aguaje: propuesta para una iniciativa. Iquitos. IIAP.

(3) Kahn, F. (1996). L' Alimentation en forệt tropicale. Vol. II. 261-274. Paris. UNESCO.

(4) Khan, F. (1988). Ecology of economically important palms in Peruvian Amazonía. Advances in Economic Botany 6, 42-49.

(5) Padoch, C. (1988). Aguaje (Mauritia flexuosa L.f.) in the economy of Iquitos, Peru. Advances in Economic Botany 6, 214-224.

(6) Pinedo-Vásquez, M. (1992). Community forest in the Peruvian Amazon: a local alternative for sustainable use of tropical forests. Advances in Economic Botany. 9, 79-86.

(7) Ruiz-Murrieta, J.; Levistre-Ruiz, J. (1966). L' Alimentation en forệt tropicale. Vol. II. 1249-1264. Paris. UNESCO.

(8) Ruiz-Murrieta, J. (1991). Food from the forest. Journal of Tropical Forest Science. 5, 115-129

(9) Ruiz-Murrieta, J.; Pinzón Rueda, R, M. Eds. (1995). Extractive Reserves. IUCN, Gland, Switzerland and Cambridge, UK.

(10) Salazar, A y Rossel, J. (1977). Estudio de la potencialidad industrial del aguaje. Lima. Proyecto ITINTEC 3102 UNA-IIA. 